\title{
Laser induced enhancement of dichroism in supported silver nanoparticles deposited by evaporation at glancing
} angles

\author{
(Ed: Glyn Lewis) \\ Ascii/Word/NAN/ \\ nano441251/PAP \\ File name \\ Date req \\ Artnum \\ Cover date
}

A Nicolas Filippin ${ }^{1}$, Ana Borras ${ }^{1}$, Victor $\mathrm{J} \mathrm{Rico}^{1}$, Fabian Frutos ${ }^{2}$ and Agustin R González-Elipe ${ }^{1}$

${ }^{1}$ Nanotechnology on Surfaces Laboratory, Materials Science Institute of Seville (ICMSE, CSIC-US), C/Americo Vespucio 49, 41092 Seville, Spain

${ }^{2}$ Applied Physics Department, E.T.S. Ingenieria Informatica (University of Seville),

Avd. Reina Mercedes s/n, 41012 Seville, Spain

E-mail: anaisabel.borras@icmse.csic.es

Received 30 July 2012, in final form 16 November 2012

Published

Online at stacks.iop.org/Nano/23/000000

\begin{abstract}
Silver nanoparticles (NP) depicting well defined surface plasmon resonance (SPR) absorption were deposited on flat substrates by physical vapor deposition in a glancing angle (GLAD) configuration. The particles were characterized by scanning electron microscopy (SEM) and atomic force microscopy (AFM) and their optical properties examined by UV-vis absorption spectroscopy using linearly polarized light. It was found that, depending on the amount of deposited silver and the evaporation angle, part of the 'as-prepared' samples present NPs characterized by an anisotropic shape and a polarization dependent SPR absorption and different colors when using polarized white light at $0^{\circ}$ and $90^{\circ}$. Low-power irradiation of these materials with an infrared Nd-YAG nanosecond laser in ambient conditions produced an enhancement in such dichroism. At higher powers, the dichroism was lost and the SPR bands shifted to lower wavelengths as a result of the reshaping of the silver NPs in the form of spheres. The possible factors contributing to the observed changes in dichroism are discussed. S] Online supplementary data available from stacks.iop.org/Nano/23/000000/mmedia
\end{abstract}

(Some figures may appear in colour only in the online journal)

\section{Introduction}

Metal nanoparticles (NPs) with surface plasmon resonance (SPR) activity have been widely studied because of their applications for the fabrication of optical devices such as filters, non-linear optical components, Raman enhancers and others [1-4]. The dichroic activity of anisotropic metal aggregates has been exploited for the development of optical filters, new encryption processes, the fabrication of polarized light emitters or materials with enhanced IR luminescence [5-8]. Dichroism, usually achieved by managing the shape and distribution of the metal aggregates along a preferential direction, can be produced by different methods. For example, by manufacturing assemblies of parallel stripes of NPs onto preformed surfaces presenting 1D periodic roughness $[6,7]$ or by more sophisticated approaches consisting of the application of soft lithographic techniques to accurately tailor the nanostructure of the materials according to asymmetric patterns [5]. A well-known soft lithographic technique widely used to modify the shape and structure of NPs relies on the use of laser scanning [9-13], either by in situ [9] or ex situ [10-13] treatments.

Recently, we have prepared dichroic structures of silver by sputtering this metal onto $\mathrm{SiO}_{2}$ thin films consisting of 
bundled arrangements of $\mathrm{SiO}_{2}$ nanocolumns (i.e., $\mathrm{Ag} / \mathrm{SiO}_{2}$ systems) prepared by glancing angle deposition (GLAD) and a posterior treatment with a nanosecond laser [14, 15]. In that case an anisotropic distribution of silver in the form of stripes was induced by a surface template effect of the nanocolumn bundles existing in the $\mathrm{SiO}_{2}$ GLAD thin films. The anisotropy was enhanced by the partial melting and agglomeration of the silver aggregates under the action of laser irradiation. The principal achievements of that work were the tuning of the SPR along the visible range and the demonstration of the applicability of the system for optical nano-patterning applications. Enhancement of dichroism by laser irradiation in deposited silver by laser is rather unusual since the most common behavior of deposited NPs consists of the agglomeration of the smaller particles into spherical and larger ones [16-18].

In the present work we essayed the preparation of silver NPs by direct evaporation at glancing angles (GLAD-PVD) by evaporation of silver in high-vacuum conditions from a point source. As will be demonstrated below, the technique provides a straightforward way to the formation of asymmetric nanoparticles with different plasmon wavelengths associated to their shape. The growth of such particular NPs is due to the specific geometry of the deposition. Different experiments shown in this work demonstrate the relation between the geometry of the deposition, the morphology and, therefore, optical properties of the nanoparticles. Meanwhile in the previous references $[14,15]$ the silver deposition was carried out by a plasma related technology, sputtering dc from a silver thread in Ar pressure. The isotropic character of the DC sputtering produces at low thickness rounded nanoparticles. On other hand, [14, 15] show the formation of elongated silver nanoparticles using a $\mathrm{SiO}_{2}$ template fabricated by GLAD. Therefore, the main differences between this protocol and the previous ones are: (1) the methodology for the preparation of the nanoparticles; (2) this new methodology allows us to further control the optical properties of the starting material, i.e. before the laser treatment; (3) this is a template-free protocol, meaning the interesting results on control of the dichroism and plasmon resonance wavelength that we demonstrated previously can be applied in a one-step routine.

This technique has been widely used for the preparation of nanocolumnar porous thin films of metals [19, 20] oxides [21-23] or semiconductor [24, 25] materials. Silver nanocolumnar films have also been obtained by this method [26-29]. Herein, we have stopped the deposition process before the development of the nanocolumns and obtained discrete silver NPs at the initial stages of growth We show that these metal nuclei have an elongated shape and present a dichroic optical behavior that can be enhanced by laser irradiation. Besides dealing with the preparation of the silver deposits and studying their optical behavior before and after the laser treatment, we discuss the implications of the GLAD geometry for the fabrication of anisotropic silver NPs on flat substrates and the factors that contribute to increase their anisotropy by laser irradiation.

\section{Experimental details}

Silver evaporation was carried out in an electron bombardment evaporator using a crucible with silver as a target. Pieces of a $\mathrm{Si}(100)$ wafer with a size of $2.5 \times 2.5 \mathrm{~cm}^{2}$ were used as substrates for the SEM characterization of the as-grown samples. Simultaneous evaporation was also carried out on glass and quartz plates that thereafter were examined by UV-vis absorption spectroscopy and underwent the laser treatments. Deposition was performed at room temperature in vacuum (i.e., $10^{-6}$ Torr) by placing the substrates at different glancing zenithal angles $\alpha$ from $60^{\circ}$ to $85^{\circ}$. The layer thickness was controlled by monitoring the evaporation process with a quartz crystal monitor (QCM) previously calibrated by comparing its response with the thickness of Ag films prepared at normal geometry. When the text refers to the amount of evaporated silver, the data always indicate the nominal thickness measured by the (QCM) place at normal geometry. Therefore, the typical cosine rule should be applied to estimate the amount of silver actually deposited on the substrates placed at glancing angles with respect to the source. For comparative purposes, samples were also prepared in a normal configuration $\left(\alpha=0^{\circ}\right)$ or at a glancing angle while rotating the substrate. In the text, samples will be named by indicating their nominal thickness followed by the deposition angle (e.g., $50 \mathrm{~nm} / 85^{\circ}$ means a sample with a nominal thickness of $50 \mathrm{~nm}$ prepared at $85^{\circ}$ zenithal evaporation angle). The samples were fabricated at deposition rates between 0.5 and $1.5 \AA \mathrm{s}^{-1}$ in all cases. The as-prepared samples were kept in a desiccator under controlled conditions until their use.

Laser post-treatment was performed at room temperature with a $20 \mathrm{~W}$ diode-pumped Nd:YAG (Powerline E, RofinBaasel Inc.) unpolarized laser emitting at $1064 \mathrm{~nm}$ with a $100 \mathrm{~ns}$ pulse width and a $20 \mathrm{kHz}$ repetition rate. The samples were scanned with a $60 \mu \mathrm{m}$ spot, measured by analyzing the laser marks on a silver thick film at the working distance (figure $\mathrm{S} 1$ at the supplementary data section available at stacks.iop.org/Nano/23/000000/mmedia), at $100 \mathrm{~mm} \mathrm{~s}^{-1}$ speed with one pulse per spot fired into the material. The maximum energy fluence applied in these experiments is $21.2 \mathrm{~J} \mathrm{~cm}^{-2}$ and was calculated as the ratio energy per pulse to area. The full width of the beam at half its maximum intensity (FWHM) supplied by the constructor is $10 \mathrm{~nm}$. UV-vis absorption spectra were recorded in a Cary 100 spectrophotometer at normal incidence and in the [220-900 nm] range with a $1 \mathrm{~nm}$ monochromator step spectrometer using linearly polarized light at $0^{\circ}$ and $90^{\circ}$.

The particle size and shape of the silver NPs evaporated on a silicon wafer were analyzed by field emission scanning electron microscopy (FESEM) in a Hitachi S4800 microscope by examining both their surfaces and cross sections. The surface topography of the deposited particles was analyzed by atomic force microscopy (AFM) in a Dulcinea microscope from Nanotec (Madrid, Spain) working in tapping mode and using high-frequency cantilevers. AFM images, taken on a $1 \times 1 \mu \mathrm{m}^{2}$ surface area, were processed with the WSxM free available software from Nanotec [30]. Besides a conventional 


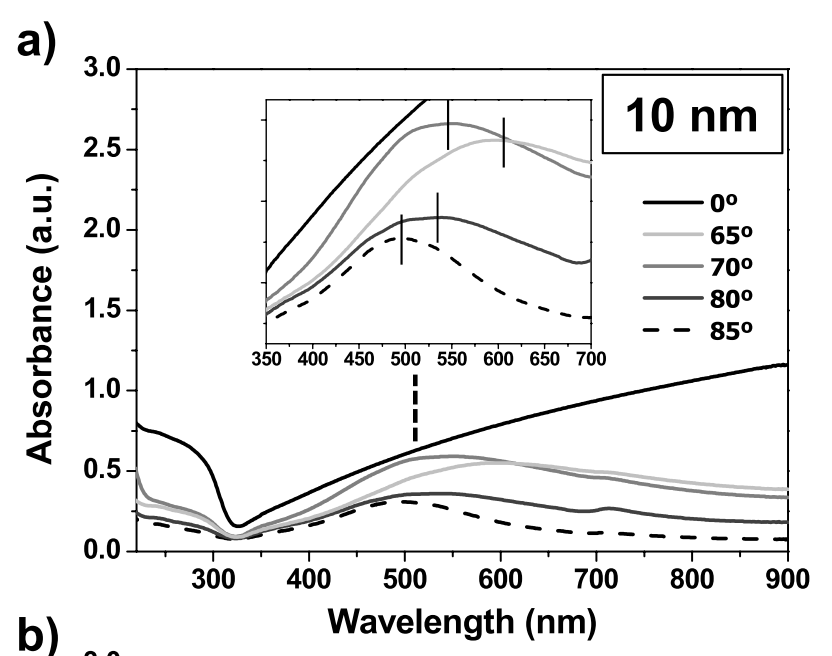

b)

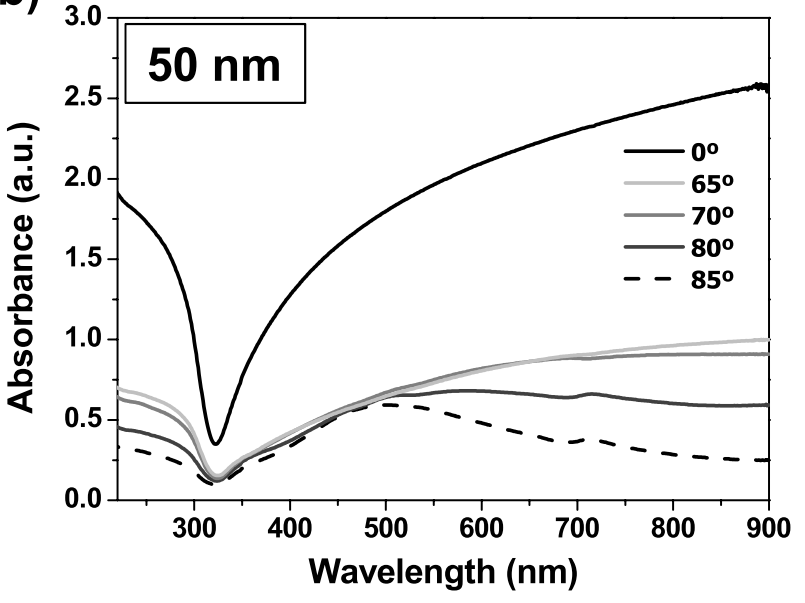

Figure 1. Absorbance spectra recorded for silver layers with (a) 10 and (b) $50 \mathrm{~nm}$ nominal thickness deposited on a glass slide at zenithal angles of $0^{\circ}, 65^{\circ}, 70^{\circ}, 80^{\circ}$ and $85^{\circ}$. The inset in panel (a) shows the changes of the absorption maxima with the deposition angle.

analysis of the surface topography, fast Fourier transforms (FFTs) of the images were calculated to assess both the shape and particle size distribution of the deposited silver.

\section{Results}

\subsection{Evaporation of silver at glancing angles}

Evaporation of silver at glancing angles on a glass or any other transparent substrate leads to the formation of optical layers characterized by either a wavelength widespread mirror reflectance or a localized SPR feature. The transition from one regime to the other depended on the amount of evaporated silver and on the zenithal evaporation angle. Figure 1 illustrates this behavior for silver deposits of 10 and $50 \mathrm{~nm}$ nominal thickness prepared at zenithal angles $\alpha=0^{\circ}, 65^{\circ}, 70^{\circ}, 80^{\circ}, 85^{\circ}$. It is apparent in this figure that the layers fabricated at the higher zenithal angles depict a defined absorption band located between 500 and $600 \mathrm{~nm}$ that we attribute to the SPR absorption of silver NPs [1-4]. At low zenithal evaporation angles, particularly at normal evaporation (i.e., $\alpha=0^{\circ}$ ), the layer behaves as a mirror without any defined absorption feature. A similar tendency was found for silver layers of intermediate nominal thicknesses of 20,30 and $40 \mathrm{~nm}$ (see the supplementary data section figure S2 available at stacks.iop.org/Nano/23/000000/ mmedia). The broad absorption between 200 and $320 \mathrm{~nm}$ presented in all the curves can be related with both light scattering in the interface with the substrates [31] and the combination of the SPR with a minimum at $\sim 310 \mathrm{~nm}$ which is characteristic of the silver systems. At such a wavelength the real and imaginary parts of the dielectric function of silver almost vanish in relation with interband transitions [32]. Different authors have also reported two absorption bands at 305 and $350 \mathrm{~nm}$ associated with the presence of $\mathrm{Ag}^{+}$and elemental Ag atoms correspondingly [33, 34]. In our case, besides the strong SPR absorption hampering the resolution of bands due to metal ions [31], we have discarded the possibility of silver oxide presence by following the absorption after an oxidation/reduction cycle and contrasting the main the strongest modification of the spectra are the displacement and narrowing of the surface plasmon resonance related to a rearrangement of the silver clusters (data not shown).

The formation of isolated silver particles at the highest zenithal angles could be confirmed by SEM observation. This analysis showed that silver deposited under these conditions aggregates in the form of small NPs with a morphology that depended on the angle of evaporation and the amount of evaporated silver. Figure 2 shows a series of normal and cross section SEM micrographs showing the characteristics of the silver grains formed in samples $50 \mathrm{~nm} / 85^{\circ}$ and $10 \mathrm{~nm} / 85^{\circ}$. This figure clearly shows that silver evaporated at zenithal angles forms small particles without completely covering the substrate. The histogram included in the figure shows that the average particle size increases with the amount of evaporated silver, as expected for a progressive percolation and agglomeration of the initial silver nuclei into larger particles. This assessment of the evolution of particle sizes, also proved for other deposition angles and equivalent thicknesses, complies with an ample series of results in the literature reporting that evaporation of small amounts of silver at normal deposition angles leads to the formation of discrete particles [35-37]. For higher amounts of evaporated silver, i.e., the conditions where a mirror-like behavior was found (cf figure 1), a homogeneous and completely percolated metal film could be observed by SEM (image not shown).

A close look at the cross section images in figures 2(c) and (d) and to the shape of the NPs in the normal images (a), (b) reveals that, even if irregular, they present a certain elongation perpendicular to the direction of evaporation. This asymmetry had a critical influence on the optical properties of these deposited particles. From now on we will use the following notation when indicating absorption for linear polarized light: linear polarized light perpendicular/parallel to the flux direction $(x) /(y)$, i.e. in the long/short axis growth of the nanoparticles (figures 2(a) and (b)), will be denoted as $0^{\circ} / 90^{\circ}$ and the corresponding SPR as longitudinal/transversal. Figure 3 shows the absorption spectra of samples $10 \mathrm{~nm} / 80^{\circ}$ and $50 \mathrm{~nm} / 85^{\circ}$ under linear polarized light. In the two cases, the most significant feature 

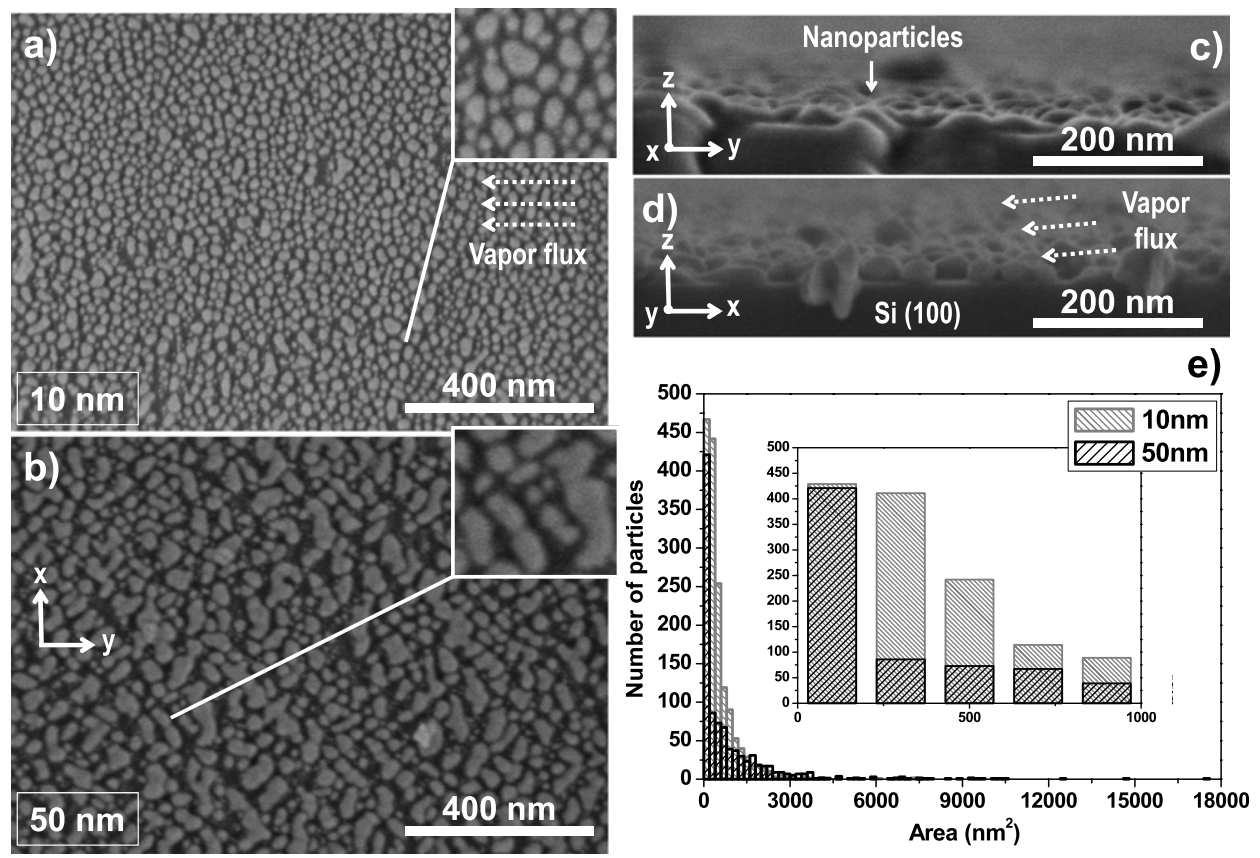

Figure 2. Normal (a), (b) and cross section (c), (d) SEM micrographs of samples $10 \mathrm{~nm} / 85^{\circ}$ (a), (c), (d) and $50 \mathrm{~nm} / 85^{\circ}$ (b). Arrows inserted in the micrographs indicate the direction of arrival of silver flux during the preparation of the samples. Coordinate axes defined with respect to this direction are also included for clarity. The insets in (a) and (b) show an enlargement of the micrograph for a better view. Cross section micrographs (c), (d) have been taken by turning the $10 \mathrm{~nm}$ sample during analysis as indicated by the coordinate axis in the figure. The histograms in (e) show the particle size distributions obtained from images (a) and (b) for each studied sample.

was the difference in the wavelength position and width of the plasmon absorption band when turning the polarizer $90^{\circ}$. This optical dichroism must result from the excitation of distinct plasmon resonances along the nanoparticles (longitudinal plasmon L-SPR) and perpendicularly to them (transverse plasmon T-SPR). The different plasmon wavelengths of the two bands indicate that the electromagnetic coupling along the nanoparticle longest dimension is stronger than along the perpendicular direction. For the two samples, the L-SPR depicts red shifts by, respectively, 56 and $84 \mathrm{~nm}$ accompanied by a broadening in the shape. Similar wavelength modifications and broadenings, although to a different extent depending on the sample, were also found for the samples that depicted a clear plasmon absorption band (table $\mathrm{S} 1$ at the supplementary data section available at stacks. iop.org/Nano/23/000000/mmedia).

At this point it is worth noting that samples prepared by GLAD while rotating the substrate or by normal evaporation and a small nominal thickness were characterized by small and rather symmetrical silver NPs and did not present any significant difference in their absorption spectra when examined with polarized light at $0^{\circ}$ and $90^{\circ}$ (see figure $\mathrm{S} 3$ available at stacks.iop.org/Nano/23/000000/mmedia). This result further supports that the optical anisotropy depicted in figure 3 has to be associated with the elongated shape of the silver NPs prepared in a GLAD configuration.

\subsection{Laser post-treatment of silver NPs prepared by GLAD}

A well-known phenomenon observed when deposited silver NPs are laser irradiated is their coarsening and agglomeration into particles of larger size [16-18]. For the anisotropic particles prepared here by GLAD, this should normally lead to the overlapping of the L-SPR and T-SPR and therefore to the decrease of the dichroism. Figure 4 shows a series of normal SEM micrographs taken for sample $50 \mathrm{~nm} / 85^{\circ}$ exposed to laser illumination with increasing laser irradiances in the range between 141 and $424 \mathrm{~kW} \mathrm{~cm}^{-2}$. This set of micrographs reveals a progressive modification of the particles from an elongated to a spherical shape and, at a first glance, the formation of larger particles when the irradiance is progressively increased. A closer look to the particle morphologies in the sample irradiated with the maximum power of the laser (see the high-magnification SEM image in figure 4(f)) shows that together with the large spherical NPs (diameters between 40 and $100 \mathrm{~nm}$ ) there are also very small spherical NPs (diameters below $10 \mathrm{~nm}$ ), thus defining a kind of bimodal (even a trimodal) distribution of particle sizes. For intermediate or the lowest irradiance conditions (i.e. 141 and $212 \mathrm{~kW} \mathrm{~cm}^{-2}$ ), although a small number of spherical NPs can be seen in the image, most of them continue having an irregular and elongated shape.

Unfortunately, the characterization of these samples by high-resolution SEM in cross section mode is hampered by charging problems associated with the use of fused silica as the substrate for the laser treatments. Nevertheless, a deeper look at the characteristics of the particles after the different irradiation experiments is achievable by AFM. The topographic images and fast Fourier transforms (FFTs) for sample $50 \mathrm{~nm} / 85^{\circ}$ presented in figure 5 confirm the previous assessment on the evolution of particle size and shape derived from the SEM analysis. Thus, the evolution of the topography 

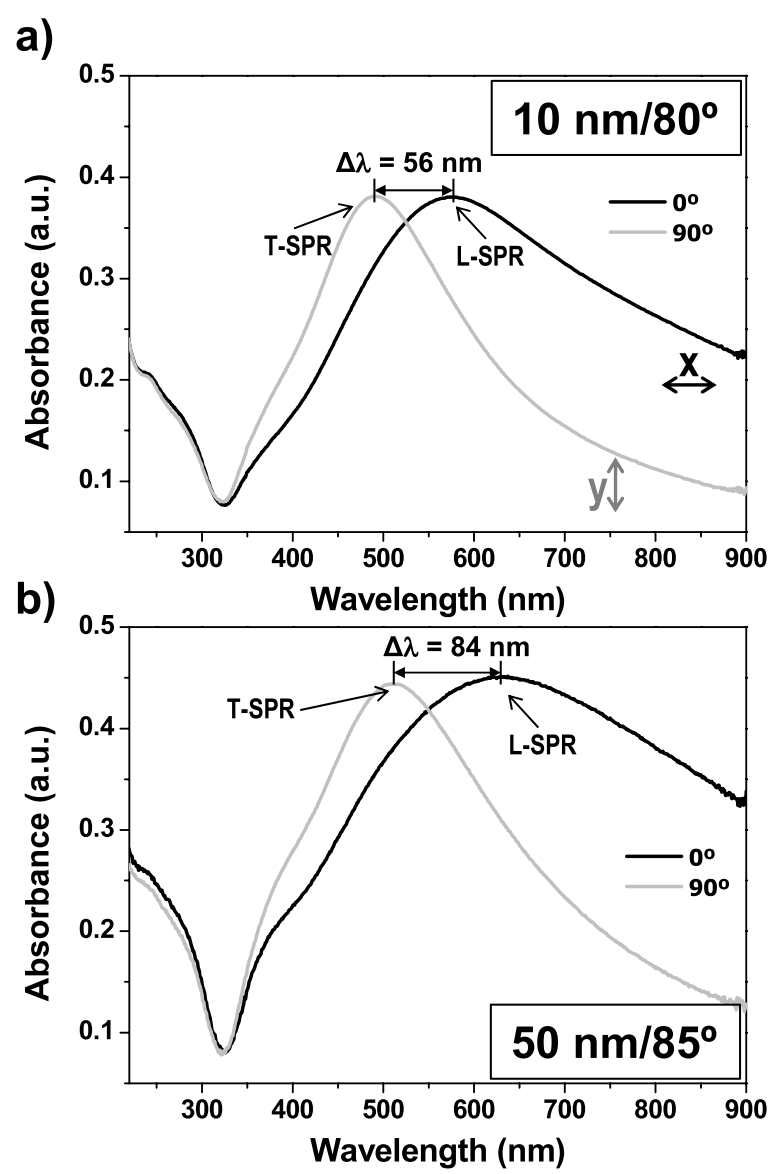

Figure 3. Absorption spectra recorded with linearly polarized light at $0^{\circ}$ and $90^{\circ}$ for samples $10 \mathrm{~nm} / 80^{\circ}$ (a) and $50 \mathrm{~nm} / 85^{\circ}$ (b).

of the samples (see the gray intensity scale in panels (a)-(c) and the histograms in panel (e)) agrees with an increase in the particle height associated with the formation of cylindrical and/or rounded particles of larger size. Meanwhile, the histograms included in the same figure clearly indicate that both the average particle size and the width of the particle size distribution increase with the irradiance, in agreement with the SEM observation of these samples in figure 4. The RMS roughness values collected in figure 5(d) confirm these tendencies. Similarly, the FFTs included in the figure provide additional evidence of the coarsening of the silver NPs when subjected to laser irradiation. For the original sample the FFT diagram is characterized by a relatively large and rectangular area which agrees with the presence of small particles with elongated shape. Increasingly higher laser irradiances produce a decrease in the size of the FFT diagram and its transformation into a symmetric pattern, both features agreeing with the formation of spherical silver particles. Thus, the height histograms demonstrate the increment in the height out of plane of the NPs with the laser treatment, meanwhile the FFT devoted to the in-plane features show the transformation into rounder nanoparticles.

The changes experienced by the morphology and size of the silver particles upon laser irradiation had a direct correlation to the optical behavior of samples. A first effect is observed in the wavelength position of the L-SPR maximum, which could be tuned along the visible range as a function of the irradiance parameter. A direct consequence of such a tuning for a given sample was the modification of its dichroism. A clear example of this effect is reported in figure 6 , showing the transmittance spectra recorded with linearly polarized light at $0^{\circ}$ (L-SPR) and $90^{\circ}$ (T-SPR) for sample $50 \mathrm{~nm} / 85^{\circ}$ subjected to increasingly higher laser irradiances. The as-grown sample presents a L-SPR at $\sim 570 \mathrm{~nm}$ that splits into two peaks, one well defined at higher wavelengths $(720 \mathrm{~nm})$ and a another one at $\sim 550 \mathrm{~nm}$. The former is also observed with very little intensity in the T-SPR spectrum, which, otherwise, remained almost unmodified. A similar effect was described and modeled in a previous work regarding the ns-laser treatment of silver nanostripes deposited on $\mathrm{SiO}_{2}$ nanocolumns [15]. At medium irradiances from 177 to $248 \mathrm{~kW} \mathrm{~cm}{ }^{-2}$, the $720 \mathrm{~nm}$ band in the L-SPR spectrum shifted to the red and merged with the component at around $550 \mathrm{~nm}$. Except for the removal of the shoulder at $720 \mathrm{~nm}$, little change can be observed in the T-SPR spectra for this range of irradiances. Treatment under higher irradiances from 318 to $424 \mathrm{~kW} \mathrm{~cm}^{-2}$ progressively transformed the plasmon contributions recorded with the two polarizations of light into a unique complex band appearing at around $400 \mathrm{~nm}$ for the maximum power of the laser. In this final band, up to three different features can be distinguished in the spectra recorded either for $0^{\circ}$ or $90^{\circ}$ polarizations.

Resulting from these changes in the absorption spectra, the color appearance of the sample illuminated with polarized white light changed as a function of both the laser irradiance and the polarization of light as reported in figure 6(c). Turning the polarizer, the full color scale $\left(0^{\circ}\right)$ obtained when exciting the L-SPR turns almost monochromatic at $90^{\circ}$. The wide variety of colors and its dependence on the polarization of light opens the possibility to create a full pallet of polarized dependent colors based on the plasmon absorption of silver NPs. Due to the focusing properties of the laser, the previous colors could be produced according to given patterns and be used for optical encoding [38, 39].

The behavior of sample $50 \mathrm{~nm} / 85^{\circ}$ described in figure 6 was similarly reproduced by all the samples where an optical anisotropy was already apparent in the 'as-prepared' films (cf table S1 at the supplementary data available at stacks. iop.org/Nano/23/000000/mmedia). In all cases, irradiation at the minimum laser irradiance produced a shift to longer wavelengths in the position of the plasmon band recorded with the $0^{\circ}$ polarized light. As a consequence, the difference in the SPR position recorded with $0^{\circ}$ and $90^{\circ}$ polarized light increased, leading to a maximum difference in the SPRs and, hence, in the color appearance of the samples when examined with white light (cf figure 6 (right)). Laser irradiation at intermediate powers produced in all cases a progressive diminution of the plasmon shift that decreased smoothly to zero at the maximum irradiance. A maximum shift of $200 \mathrm{~nm}$ was obtained for sample $50 \mathrm{~nm} / 85^{\circ}$. To summarize the effect of the laser on the different samples, figure 7 shows a plot of the magnitude of the plasmon shifts between the SPR maxima obtained with $0^{\circ}$ and $90^{\circ}$ linearly polarized light when the different samples were subjected to laser 

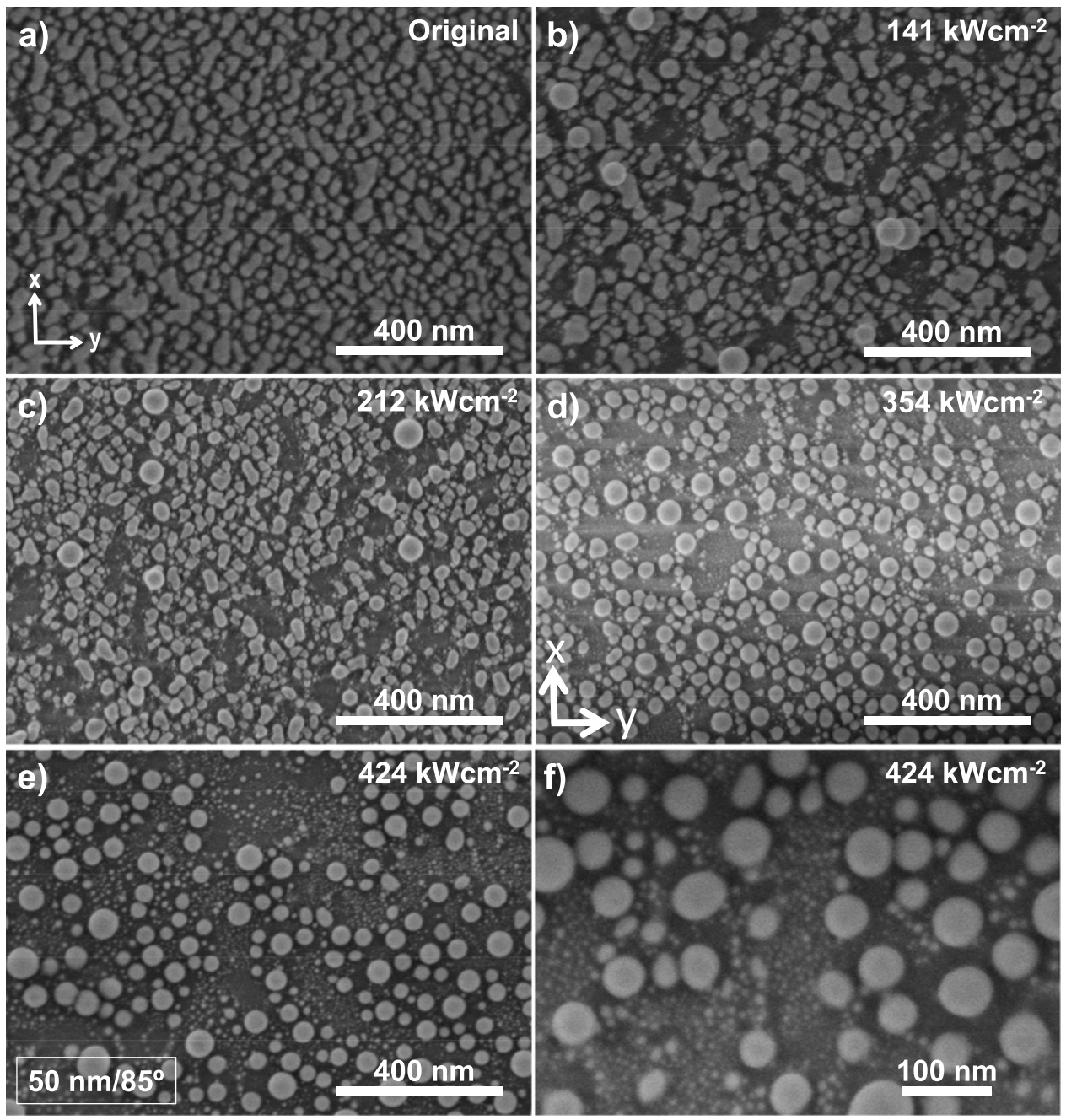

Figure 4. Normal view SEM micrographs of sample $50 \mathrm{~nm} / 85^{\circ}$ subjected to increasingly higher laser irradiances as indicated in the images from (a) to (f). Panel (f) shows a higher magnification micrograph to better observe the heterogeneous distribution of particle sizes present in the sample after treatment at the highest irradiance conditions.

irradiation at increasingly higher irradiances. It must be noted that practically no anisotropy enhancement was obtained for samples prepared at normal evaporation or by rotating the substrate during evaporation in a GLAD configuration that, as previously indicated, did not present a noticeable anisotropy in the 'as-prepared' state.

\section{Discussion}

\subsection{GLAD formation of silver NPs}

Growth of silver nanocolumns by glancing deposition is known to yield a very interesting optical phenomenology, widely studied since the 1980s [26-29]. In contrast, the initially formed particles deposited at glancing angles and their modification by laser irradiation has not received much attention. Our results have shown that silver NPs prepared by evaporation in a GLAD configuration present optical anisotropy, likely linked with their elongated shape, with the longest dimension perpendicular to the direction of evaporation. Asymmetric growth of GLAD microstructures is a common phenomenon in thin films that sometimes becomes apparent by the agglomeration of the individual nanocolumns in the form of 'bundles' $[35,40]$. The asymmetric NP shape obtained here must be a first indication of this tendency to form anisotropic structures already at the initial nucleation steps. Our results have also shown that a plasmonic structure appears for certain angles of evaporation and relatively low amounts of evaporated metal. For other preparation conditions with a higher amount of silver, the silver layer presents a mirror-like behavior where no SPR can be detected. We attribute this result to the percolation of the silver into a continuous structure where no isolated particles can be differentiated. We have found that the elongated particles prepared at GLAD present a dichroic behavior when examined with linearly polarized light that is not found when evaporated in a normal geometry [35-37]. This dichroism and its evolution upon laser irradiation must be a result of the change of the morphological characteristics of the NPs. 

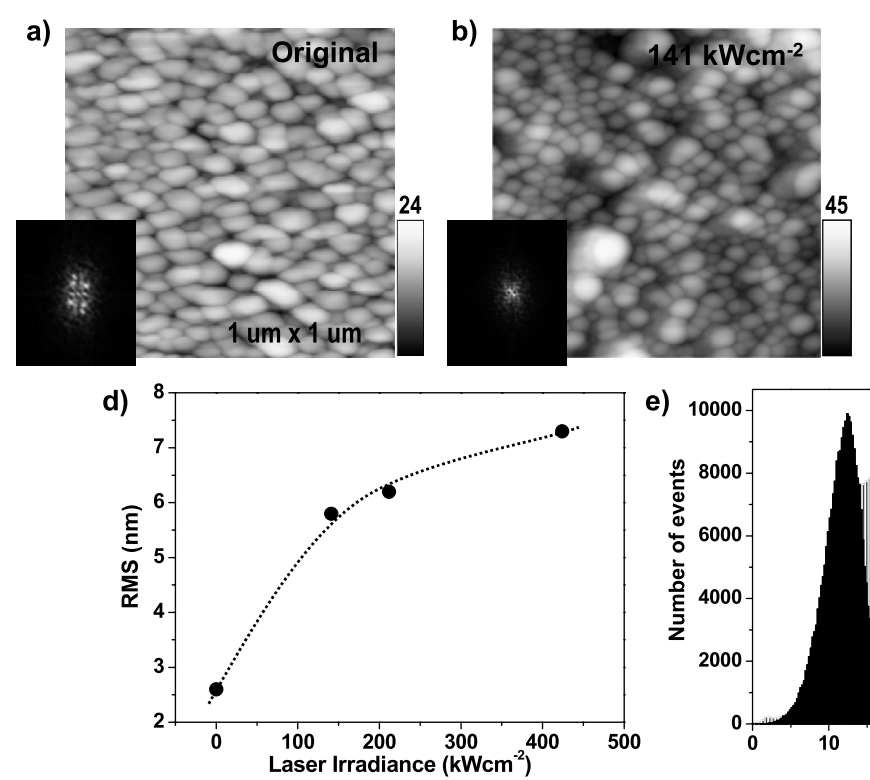
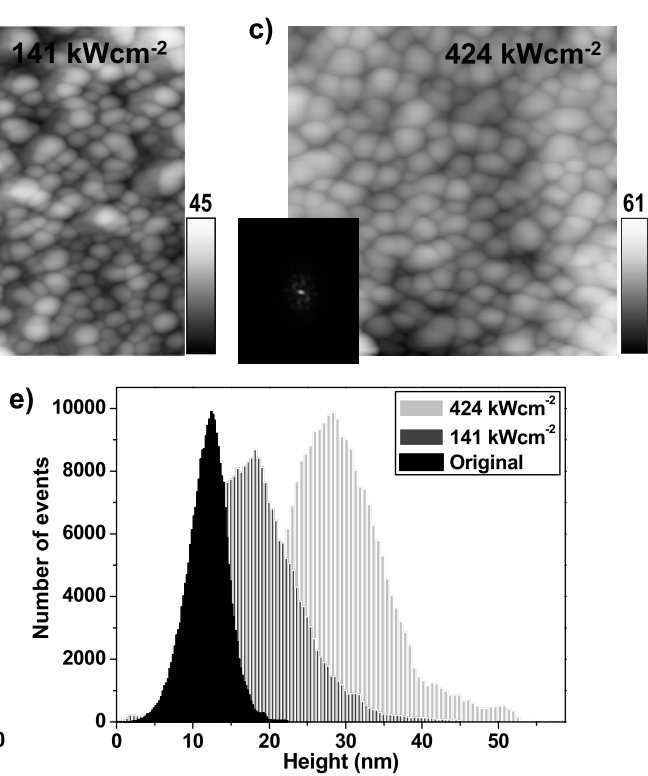

Figure 5. (a)-(c) AFM topographic images and Fourier transforms (FFTs) as insets of sample $50 \mathrm{~nm} / 85^{\circ}$ as prepared by GLAD and after being subjected to increasingly higher laser irradiances as indicated; (d) RMS roughness as a function of the laser irradiance; (e) height histograms of the representative images in (a)-(c).

a)

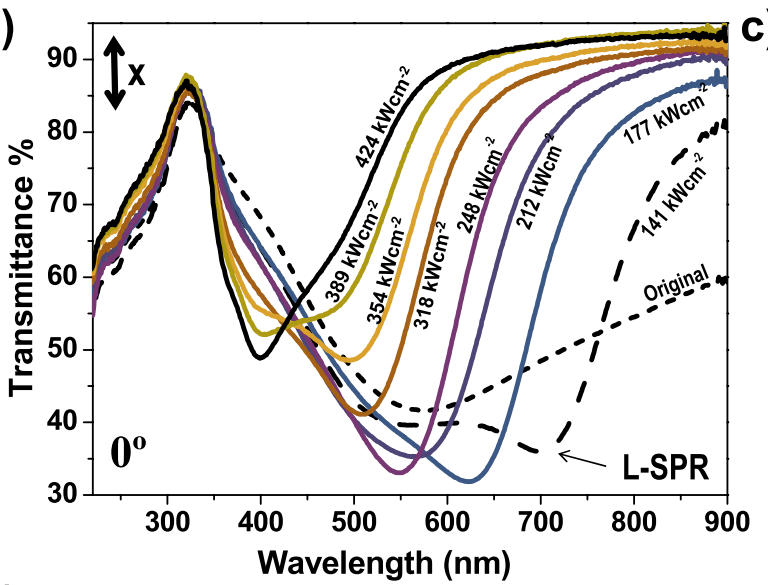

b)

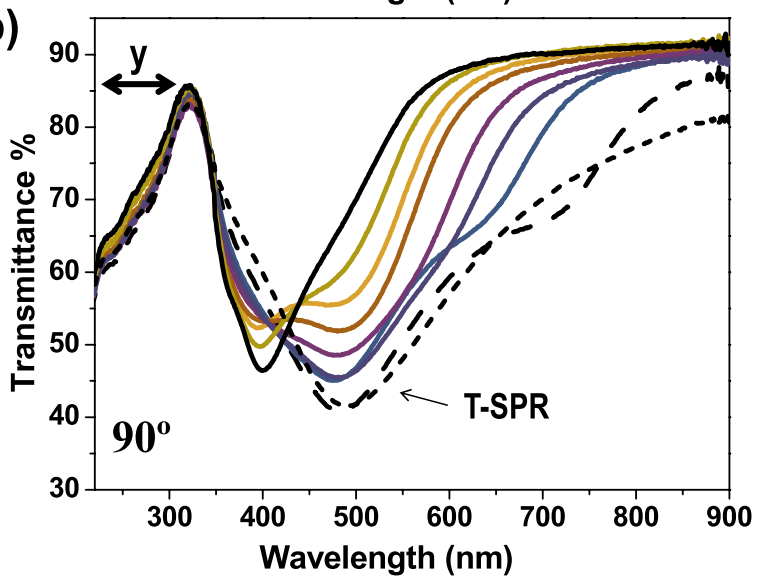

c)
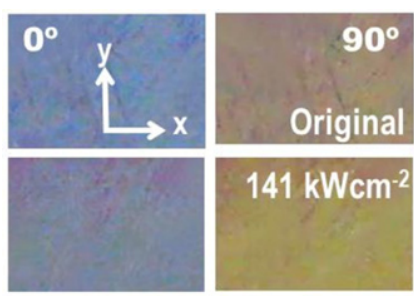

$177 \mathrm{kWcm}^{-2}$
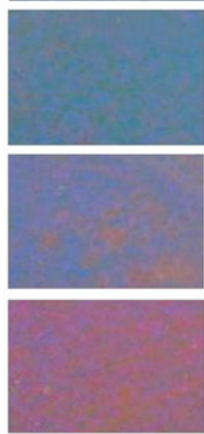

$248 \mathrm{kWcm}^{-2}$
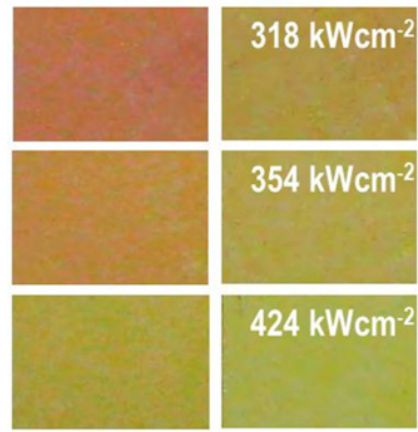

Figure 6. Transmission spectra recorded with linearly polarized light at $0^{\circ}$ (a) and $90^{\circ}$ (b) for sample $50 \mathrm{~nm} / 85^{\circ}$ subjected to laser treatments with increasing irradiances between 141 and $424 \mathrm{~kW} \mathrm{~cm}^{-2}$. The color code is the same in both graphs. (c) Color photographs taken for the same samples illuminated with polarized $\left(0^{\circ}\right.$ and $\left.90^{\circ}\right)$ white light showing the production of a color scale when exciting the longitudinal plasmon. 


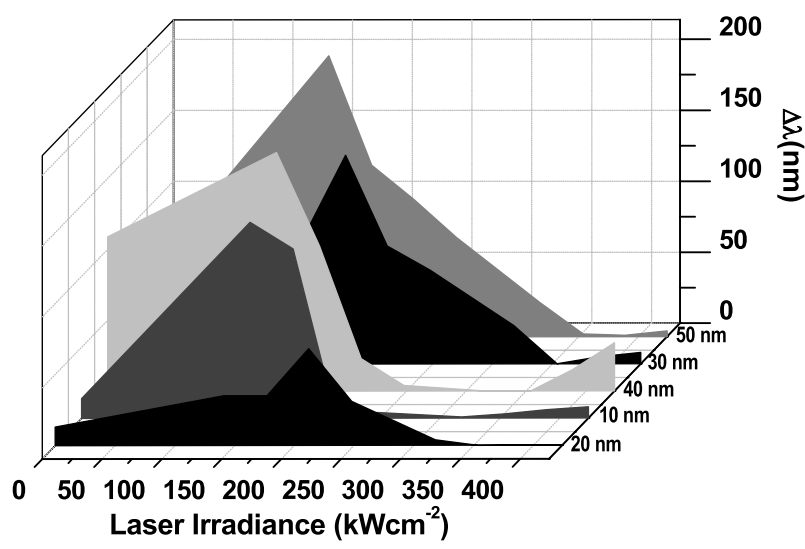

Figure 7. Evolution of the SPR shift between the UV-vis spectra recorded with $0^{\circ}$ and $90^{\circ}$ polarized light for the different studied samples treated with increasingly higher laser irradiances.

\subsection{Laser irradiation of elongated silver NPs}

In general, deposited silver NPs subjected to laser irradiation tend to agglomerate into larger particles with spherical shape [16-18], producing a red shift in the position of the plasmon. The observed optical behavior found when our samples were subjected to low laser irradiances was more complex and resulted in an enhancement in the optical anisotropy when using polarized light. In the 'as deposited' state the NPs prepared at glancing angles appear to be rather flat (cf, figure 2(c)) and elongated in the direction to the arrival of the incoming flux of silver during evaporation. This morphology must be the cause of the different shape of the L-SPR and T-SPR spectra and the observed shift in their maxima. According to the literature [41, 42], among the factors contributing to the red shift in the position of the plasmons we can quote the elongated aspect ratio, the interaction with the substrate and the irregular shapes with relatively sharp corners in these NPs.

Laser irradiation produces a progressive change in both the T-SPR and L-SPR modes that must be accounted for by some modifications in the morphology of the NPs. The pictorial model presented in figure 8 attempts to illustrate the morphological changes of the silver NPs evidenced by the SEM and AFM analysis of the original and laser irradiated samples (cf, figures 4 and 5). According to it, evaporation at glancing angles leads to the growth of elongated particles of different sizes and shapes but with a preferential orientation of their longest dimension perpendicular to the evaporation direction (figure 8(a)). The actual averaged anisotropy of the evaporated particles depends on both the amount of evaporated material and the zenithal angle of deposition, as supported by the plasmon shifts reported in table $\mathrm{S} 1$ (available at stacks.iop.org/Nano/23/000000/mmedia). According to the optical characterization results summarized in this table, when the deposited amount of silver increases, percolation of the NPs takes place (figure 8(b)) and the SPR absorption becomes replaced by a continuous and intense reflection behavior. Our results have also shown that the rather scattered distribution of particle sizes characteristic of the GLAD samples (cf figure 2(b)) can be modified by laser irradiation. At the lowest irradiance values, the initial broad L-SPR band splits into two, with one part shifted to longer wavelengths (cf, figure 6(a)). According to figure 8(c), we attribute these changes to the preferential merging of the smallest particles along the longest axis of the largest ones and to a change in the edge sharpness,

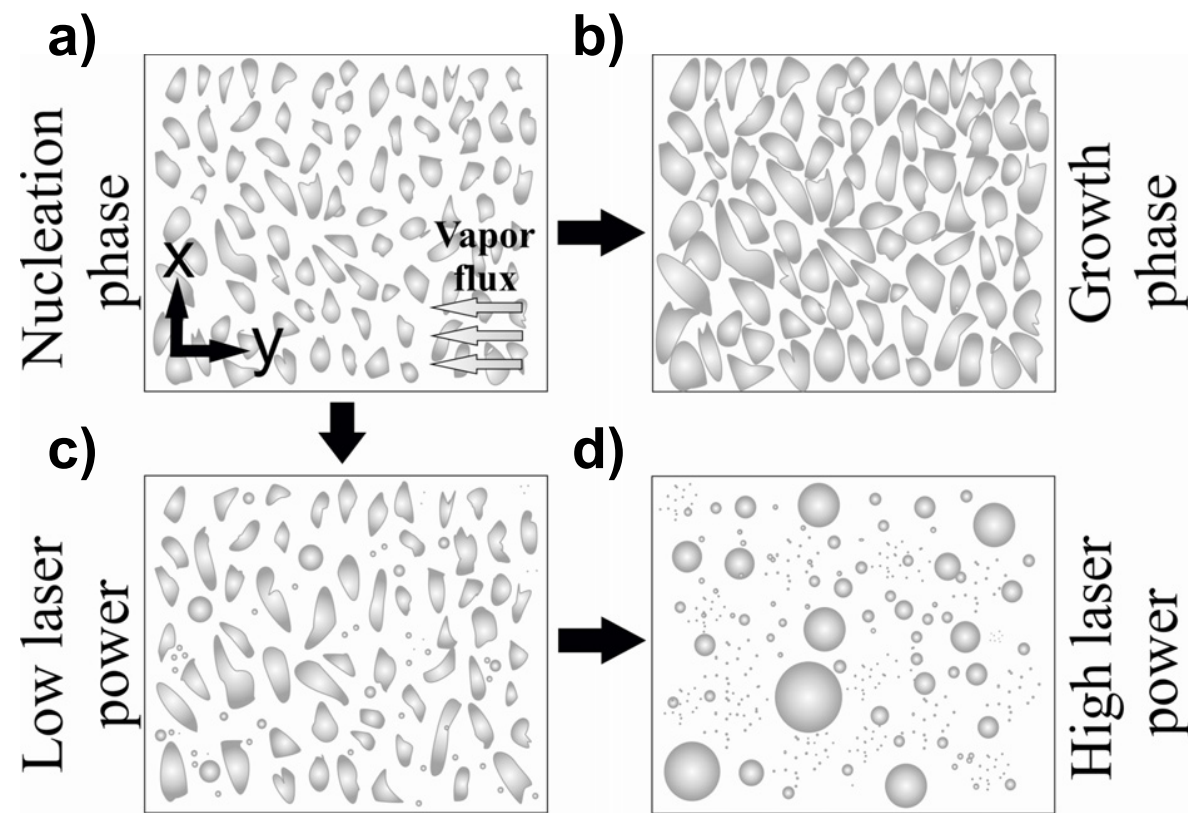

Figure 8. Scheme showing the evolution of particle size and shapes upon laser irradiation of the silver NPs prepared under the GLAD configuration. (a) Elongated silver NPs grown by GLAD. (b) Percolation of the NPs to form a continuous granular film. (c) Effect of low laser irradiances on the NP size and shapes. (d) Effect of high laser irradiances on the NP size and shapes. 
and likely the planarity of the resulting particles. The sum of all these effects would produce an additional increase in the optical anisotropy of the system and lead to an enhancement of the dichroism.

At medium irradiance values the T-SPR and L-SPR maxima shift continuously to shorter wavelengths while the optical anisotropy is progressively lost with the value of this parameter (cf, figures 6(a) and (b)). In the final situation, the irradiated samples have lost their optical anisotropy and their spectra are characterized by a complex band at around d $400 \mathrm{~nm}$ where up to three different components can be differentiated. This progressive change can be attributed to a progressive detachment of the particles from the surface and the adoption of a spherical shape (figure $8(\mathrm{~d})$ ). A partial melting of the silver aggregates, in line with previous studies on ns-laser interaction with metallic silver [36, 43] can be claimed to justify these results. In agreement with figures 4(e) and (f), this final situation would be characterized by a wide distribution of quasi-spherical particles sizes, thus accounting for the three features observed in the transmission spectra of the highly irradiated samples. It is worth stressing that even if on average no large variations in particle sizes exist between the 'as-prepared' and the highly irradiated samples, large changes in the SPR maxima and the development of dichroism appear in the GLAD grown and mildly laser treated NPs. The aforementioned factors of NP high aspect ratio, interaction with the substrate and/or the development of sharp corners [41, 42] would be the factors contributing to both the initial enhancement of dichroism at low laser irradiances and its removal and red shift in the position of the plasmon maxima after irradiating with high laser powers.

\section{Conclusion}

In the previous results and discussion we have shown that silver NPs prepared by evaporation at glancing angles depict a strong dichroism that can be enhanced by a mild laser irradiation. We attribute these optical effects to the formation of flat and elongated NPs when the evaporation is carried out along this geometry and to the modification of these morphological characteristics when the prepared samples are treated with a laser. By controlling the amount of deposited material, the evaporation angle and the laser irradiance we have been able to prepare a large set of dichroic color patterns. The simplicity of the method, not requiring any template, 1D periodic roughness effect of the substrate or the use of complex lithographic techniques, and its compatibility with any kind of substrate material are some of the most advantageous features of the procedure. Its use for optical encoding by moving the laser beam along certain predefined patterns is likely one of the applications of the developed technology with greater potential.

\section{Acknowledgments}

We thank the Junta de Andalucía (Projects P09-CTS-5189, TEP5283 and FQM-6900) and the Ministry of Economy and Competitiveness (Projects CONSOLIDER CSD200800023, MAT2010-21228, and MAT2010-18447) for financial support.

\section{References}

[1] Hutter E and Fendler J H 2004 Adv. Mater. 161685

[2] Giannini V, Fernandez-Domínguez A I, Heck S C and Maier S A 2011 Chem. Rev. 1113888

[3] Liang H Y, Li Z P, Wang W Z, Wu Y S and Xu H X 2009 Adv. Mater. 214614

[4] Rycenga M, Cobley C M, Zeng J, Li W, Moran Ch H, Zhang Q, Qin D and Xia Y 2011 Chem. Rev. 1113669

[5] Mertens H and Polman A 2006 Appl. Phys. Lett. 89211107

[6] Camelio S, Babonneau D, Lantiat D, Simonot L and Pailloux F 2009 Phys. Rev. B 80155434

[7] Fort E, Ricolleau C and Sau-Pueyo J 2003 Nano Lett. 365

[8] Suzuki M, Maekita W, Kishimoto K, Teramura S, Nakajima K, Kimura K and Taga Y 2005 J. Appl. Phys. 44 L193

[9] Cavallini M, Albonetti C and Biscarini F 2009 Adv. Mater. 211043

[10] Wenzel T, Bosbach J, Goldmann A, Stietz F and Träger F 1999 Appl. Phys. B 69513

[11] Kaempfe M, Graener H, Kiesow A and Heilmann A 2001 Appl. Phys. Lett. 791876

[12] Link S and El-Sayed M 1999 J. Phys. Chem. B 1038410

[13] Zijlstra P, Chon J W M and Gu M 2009 Nature 409410

[14] Sanchez-Valencia J R, Toudert J, Borras A, Lopez-Santos C, Ortega-Feliu I, Barranco A and Gonzalez-Elipe A R 2010 Plasmonics 5241

[15] Sanchez-Valencia J R, Toudert J, Borras A, Barranco A, Lahoz R, de la Fuente G F, Frutos F and Gonzalez-Elipe A R 2011 Adv. Mater. 23848

[16] Nouneh K, Oyama M, Lakshminarayana G, Kityk I V, Wojciechowski A and Ozga K 2011 J. Alloys Compounds $\mathbf{5 0 9} 9663$

[17] Ebothe J, Miedzinski R, Reshakb A H, Nounehd K, Oyamad M, Aloufye A and El Messiry M 2009 Mater. Chem. Phys. 113187

[18] Cattaruzza E, Mardegan M, Trave E, Battaglina G, Calvelli P, Enrichic F and Gonella F 2011 Appl. Surf. Sci. 2575434

[19] Tawfick S, De Volder M, Copic D, Park S J, Oliver C R, Polsen E S, Roberts M J and Hart A J 2012 Adv. Mater. 241628

[20] Messier R, Venugopal V C and Sunal P D 2000 J. Vac. Sci. Technol. A $\mathbf{1 8} 1538$

[21] Gonzalez-Garcia L, Lozano G, Barranco A, Miguez H and Gonzalez-Elipe A R 2010 J. Mater. Chem. 206408

[22] Sanchez-Valencia J R, Blaszczyk-Lezak I, Espinos J P, Hamad S, Gonzalez-Elipe A R and Barranco A 2009 Langmuir 259140

[23] Alvarez R, Gonzalez-Garcia L, Romero-Gomez P, Rico V, Cotrino J, Gonzalez-Elipe A R and Palmero A 2011 J. Phys. D: Appl. Phys 44385302

[24] Hawkeye M M and Brett M J 2007 J. Vac. Sci. Technol. A 251317

[25a] Van Kranenburg H and Lodder C 1994 Mater. Sci. Eng. R 11295

[25b] Hodkinson I and Wu Q H 2001 Adv. Mater. 13889

[26] Chaney S B, Zhang Z Y and Zhao Y P 2006 Appl. Phys. Lett. 89053117

[27] Liu Y, Fan J, Zhao Y P, Shanmukh S and Dluhy R A 2006 Appl. Phys. Lett. 89173134

[28] Zhao Y P, Chaney S B and Zhang Z Y 2006 J. Appl. Phys. 100063527

[29] Bloemer M J, Ferrell T L, Buncick M C and Warmack R J 1988 Phys. Rev. B 378015 
[30] Horcas I, Fernandez R, Gomez-Rodriguez J M, Colchero J, Gomez-Herrero J and Baro A M 2007 Rev. Sci. Instrum. 78013705

[31] Ferraris M, Perero S, Miola M, Ferraris S, Verne E and Morgiel J 2010 Mater. Chem. Phys. 120123

[32] Ehrenreich H and Philipp H R 1962 Phys. Rev. B 1281622

[33] Linnert T, Mulvaney P, Henglein A and Weller H 1990 J. Am. Chem. Soc. 1124657

[34] Chiaretta D, Milanese D, Menke Y, Ferraris M and Pirri F J 2006 J. Non-Cryst. Solids 3522548

[35] Mitchell C E J, Howard A, Carney M and Egdell R 2001 Surf. Sci. 490196

[36] Yang K Y, Choi K C and Won C 2009 Appl. Phys. Lett. 94173301
[37] Lee M H, Dobson P J and Cantor B 1992 Thin Solid Films 219199

[38] Siozios A, Koutsogeorgis D C, Lidorikis E,

Dimitrakopulos G P, Kehagias Th, Zoubos H,

Komninou Ph, Cranton W M, Kosmidis C and

Patsalas P 2012 Nano Lett. 12259

[39] Han R, Zhang X, Wang L, Dai R and Liu Y 2011 Appl. Phys. Lett. 98221905

[40] Vankranenburg H and Lodder C 1994 Mater. Sci. Eng. R 11295

[41] Rycenga M, Cobley C M, Zeng J, Li W, Moran C H, Zhang Q, Qin D and Xia Y 2012 Chem. Rev. 1113669

[42] Zeng J, Roberts S and Xia Y 2010 Chem. Eur. J. 1612559

[43] Link S and El-Sayed M 1999 J. Phys. Chem. B 1038410 


\section{Queries for IOP paper 441251}

Journal: Nano

Author: A Nicolas Filippin et al

Short title: Laser induced enhancement of dichroism in supported silver nanoparticles deposited by evaporation at glancing angles

\section{Page 1}

Query 1:

Author: Please check the author names and affiliations carefully.

\section{Page 1}

Query 2:

Author: Please be aware that the colour figures in this article will only appear in colour in the Web version. If you require colour in the printed journal and have not previously arranged it, please contact the Production Editor now.

\section{Page 3}

Query 3:

Author: 'contrasting the main the strongest'. It seems that some text is missing. Please modify as necessary.

\section{Page 9}

Query 4:

Author: What is ' $d$ ' given here?

\section{Page 9}

Query 5:

Author: Please check the details for any journal references that do not have a blue link as they may contain some incorrect information. Pale purple links are used for references to arXiv e-prints. 